\title{
Prospective evaluation of World Health Organization criteria to assist diagnosis of tuberculosis in children
}

\author{
K.A.F. Houwert*, P.A. Borggreven*, H.S. Schaaf+, E. Nel+, P.R. Donald+, J. Stolk*
}

\begin{abstract}
Prospective evaluation of World Health Organization criteria to assist diagnosis of tuberculosis in children. K.A.F. Houwert, P.A. Borggreven, H.S. Schaaf, E. Nel, P.R. Donald, J. Stolk. @ERS Journals Ltd 1998.

ABSTRACT: Because of the difficulty in confirming childhood tuberculosis (TB), the World Health Organization (WHO) proposes a hierarchical approach to diagnosis using history and certain clinical features to help to improve the control of TB in communities. The objective of this study was to evaluate prospectively in children the diagnostic value of recent weight loss or failure to gain weight adequately, cough or wheezing for $>2$ weeks and recent household contact with an adult case of pulmonary TB. These evaluations were performed in 627 children presenting to the paediatric outpatient department of a tertiary hospital situated in the Western Cape Province of South Africa and serving a community with a very high incidence of TB $(>1,000$ per $100,000)$. If at least one of the criteria was present, the diagnosis of $T B$ was investigated further by clinical investigation, Mantoux test, chest radiography and TB culture from gastric aspirate.

One or more of the proposed criteria for diagnosing TB in childhood were present in 206 children $(33 \%)$. TB confirmed by culture of Mycobacterium tuberculosis from gastric aspirate was found in 10 children (5\%). After diagnostic work-up, 23 children $(11 \%)$ were considered to have probable $\mathrm{TB}$, whereas $173(84 \%)$ were, after follow-up of 8 weeks, thought not to have TB. In this study the simultaneous presence of the three WHO criteria for suspecting TB had a positive predictive value of $63 \%$.

These results should assist in the more precise delineation of the predictive value of the proposed World Health Organization approach to the diagnosis of tuberculosis in childhood.
\end{abstract}

Eur Respir J 1998; 11: 1116-1120. *Dept of Pulmonology, Leiden University
Medical Center, The Netherlands. +Dept of
Paediatrics and Child Health, Tygerberg
Hospital and the University of Stellen-
bosch, South Africa.

Correspondence: J. Stolk

Dept of Pulmonology (C3-P)

Leiden University Medical Center

P.O. Box 9600

2300 RC Leiden

The Netherlands

Fax: 31715248118

Keywords: Childhood

tuberculosis

World Health Organization criteria

Received: January 71997

Accepted after revision February 71998
The tuberculosis (TB) control strategies of the World Health Organization (WHO) and the International Union Against Tuberculosis and Lung Disease are firmly focused on sputum microscopy smear-positive pulmonary TB in adults [1]. Children, nonetheless, represent a significant proportion of TB cases and childhood TB places an equally significant burden on the health services of developing countries. In 1989 it was reported that approximately $1,300,000$ cases and 450,000 deaths as result of TB occurred annually worldwide in children of $<15$ yrs of age [2, 3]. In the Western Cape Province of South Africa a very high incidence of TB is experienced (709 per 100,000 in 1994); the incidence of TB in children living in the Western Cape Province is six times higher than the national incidence rate and it was 901 per 100,000 in children $<4$ yrs of age in 1989 [4].

Traditionally, the diagnosis of TB in childhood is based on clinical criteria such as failure to thrive or malnutrition, peripheral lymphadenopathy, persistent wheeze or cough, chest radiography, history, in particular a history of close household contact with an adult with pulmonary TB and tuberculin testing [5]. In only a minority of cases is a culture of Mycobacterium tuberculosis obtained from gastric aspirate or sputum (in older children).

Children with pulmonary TB present with a wide variety of signs and symptoms. Children may either be asymp- tomatic or, at the other extreme, present with severe weight loss and wasting in cases with disseminated TB. Most children undergoing primary infection have few if any symptoms but they occasionally complain of lassitude and anorexia in combination with poor weight gain.

Childhood TB may be unrecognized and diagnosis delayed as a consequence of the lack of ready access to more sophisticated diagnostic investigation, especially in developing countries. The aim of this study was to evaluate, prospectively for the first time the criteria proposed by the WHO for suspecting TB in childhood [6]. The study was conducted in a community with a high incidence of TB and diagnostic work was performed within a tertiary care facility.

\section{Patients and methods}

\section{Subjects}

This study was conducted in the overnight holding ward of the Paediatric Outpatient Department of Tygerberg Hospital. This tertiary care hospital serves a community consisting of several suburbs, close to the city of Cape Town, with a particularly high incidence of TB $(>1,000$ 
per 100,000 in 1991) [7]. Children <13 yrs of age are admitted to this ward when a bed is not immediately available in an inpatient ward, when it is considered likely that the child will be fit for outpatient treatment within $24 \mathrm{~h}$ or when there is some diagnostic uncertainty in an ill child. All children present in the ward on weekday mornings during the period October 1995 to February 1996 were evaluated with respect to: 1) recent weight loss or failure to gain in weight; 2) cough or wheeze present for $\breve{S} 2$ weeks; and 3) close household contact with an adult with pulmonary TB or a recent history of pulmonary TB.

Close household contact with an adult with recently active (within the last 12 months) pulmonary TB was verified by contact with the local authority clinic closest to the child's home. The adults were either on anti-TB therapy because their sputum was either smear or culture positive for M. tuberculosis or had already completed their treatment. Close contacts were defined as adults with pulmonary TB living and sleeping in the same house on the same plot.

The study was approved by the Ethical Committee of the Faculty of Medicine of the University of Stellenbosch and written, informed consent was obtained from all parents or guardians for their children's inclusion in the study.

\section{Design}

Children included in this prospective study were selected from all children admitted to the holding ward of the Paediatric Outpatient Department. Selection was based on the particular attention to the provisional guidelines for diagnosis of childhood TB proposed by the WHO (table 1a). The subjects were studied for $24 \mathrm{~h}$ and a final classification into the categories confirmed TB, probable TB and no TB was made 2 months after the first visit.

\section{Methods}

Recent weight loss or failure to gain adequate weight were verified from "Road to Health" cards and hospital

Table 1. - World Health Organization provisional guidelines for the diagnosis of pulmonary tuberculosis in children [1]

a) Suspected tuberculosis

An ill child with a history of contact with a confirmed case of pulmonary tuberculosis

Any child

Not regaining normal health after measles or whooping cough

With loss of weight, cough and wheeze not responding to antibiotic therapy for respiratory disease

With painless swelling in a group of superficial nodes

b) Probable tuberculosis

A suspect case and any of the following

Positive $(>10 \mathrm{~mm})$ induration on tuberculin testing

Suggestive appearance on chest radiograph

Suggestive histological appearance of biopsy material

Favourable response to specific antituberculous therapy

c) Confirmed tuberculosis

Detection by microscopy or culture of tubercle bacilli from secretions or tissues

Identification of tubercle bacilli as Mycobacterium tuberculosis by culture characteristics and Local Authority Health Clinic records. Weight loss was defined as the loss of $>10 \%$ of previous maximum weight, and failure to gain was defined as no weight gain for a period of 2 months, the child having been weighed on at least two occasions during this period.

Cough or wheeze for $>2$ weeks was documented by taking a history from a parent or caregiver.

A history of close household contact with an adult with active pulmonary TB within the previous 12 months was verified by contact with the nursing personnel at the Local Authority Health Clinic closest to the child's home. Close household contacts were defined as adults with pulmonary TB living in the same house as the child. A diagnosis of active pulmonary TB in adults was accepted only in the presence of positive sputum microscopy for acid-fast bacilli or sputum culture for M. tuberculosis.

Children with at least one of the criteria for suspecting TB were assessed with regard to their history and clinical features. The history was taken to document previous illness and previous treatment for TB. On clinical examination the weight for age, peripheral lymphadenopathy, respiratory signs, hepatosplenomegaly and temperature were documented. A tuberculin test was performed, a chest radiograph taken and at least one early-morning gastric aspirate submitted for culture of $M$. tuberculosis by radiometric assay (Bactec, Cape Town, South Africa). Unfortunately, not all of the parents of children presenting with one or more of the criteria were prepared to remain with their child in the holding ward for completion of all of the planned investigations.

Tuberculin skin testing was by the Mantoux test with 5 units of purified protein derivative (RIVM, Bilthoven, The Netherlands). The test was administered by nursing or medical personnel who were familiar with the technique. Parents were requested to return for reading of the test after 48-72 h. In the case of children discharged for community follow-up, the result was read by Local Authority Health Clinic personnel. More than $90 \%$ of infants in the Western Cape Province receive bacille Calmette-Guérin (BCG) vaccination neonatally with the Tokyo 172 strain [8]. BCG vaccination caused skin tuberculin hypersensitivity in South Africa between 6-15 mm [9]. As there is also evidence for a small change in the level of tuberculin hypersensitivity in South Africa for up to 2 yrs following BCG vaccination, only an induration of $>15 \mathrm{~mm}$ was regarded as significant and indicative of TB infection [10].

Frontal and lateral chest radiographs were evaluated for possible primary TB by a panel of four of the authors, consisting of two consultant paediatricians with special interest in childhood TB (H.S. Schaaf and P.R. Donald), who were blinded to any clinical data. When full agreement among all of the reviewers could not be achieved with respect to the presence or absence of a radiological feature it was regarded as absent for statistical purposes. The presence of unequivocal hilar or paratracheal adenopathy, miliary TB, lobar, segmental or bronchopneumonic opacification, cavitation or pleural effusion was noted. Airway narrowing was considered to indicate the presence of adenopathy and any airtrapping and flattening of the diaphragms was noted. In this study chest radiographs were thus classified as: 1) showing unequivocal mediastinal lymphadenopathy or miliary TB; 2) having an appearance suggestive of TB (a nonresolving lobar or segmental 
opacification, cavitation or pleural effusion); 3) abnormal but having no features suggestive of TB; or 4) normal.

Human immunodeficiency virus (HIV) infection has only recently been detected in the Western Cape Province and in a 1991 survey the prevalence rate in females attending antenatal clinics was $0.37 \%$ (95\% confidence interval 0.18-0.57) [11]. HIV testing was not undertaken routinely as part of this study and was performed only in children in whom there was a clinical reason to suspect HIV infection or acquired immunodeficiency syndrome (AIDS). HIV testing was undertaken only following parental counselling and with parental consent. All HIV-positive children and their parents were referred to the Infectious Disease Clinic of the Department of Paediatrics and Child Health.

At least 2 months after entry into the study all suspect cases of TB were reclassified as having probable TB, confirmed TB or as not having TB.

Children were considered as having probable TB by: 1) the presence of a chest radiograph showing either unequivocal hilar or paratracheal adenopathy or miliary TB; or 2) a Mantoux test with an induration of Š $15 \mathrm{~mm}$. TB was considered proven by culture of $M$. tuberculosis from gastric aspirate or any other source.

\section{Analysis}

The positive predictive value of three WHO criteria was calculated from individuals with a confirmed or probable diagnosis of TB divided by the total number of patients who reported three positive WHO criteria.

\section{Results}

During the study the parents or caretakers of 627 children from several suburbs of the Tygerberg community were interviewed. At least one of the proposed criteria for diagnosing tuberculosis in childhood was present in 206 children (33\%), with a mean age of 22.7 months (sD 23.9 months). Among these children, 10 had M. tuberculosis in the gastric aspirate. Another 23 children were classified as probable TB when at least one of the criteria in table $1 \mathrm{~b}$ was present. TB could not be diagnosed in 173 children.

The number of children with the individual criteria in each of the final diagnostic categories is summarized in table 2 . The highest percentage of confirmed and probable TB cases ( 23 of the 65 children, 35\%) was found amongst the children in close household contact with adult pulmonary TB. However, 42 children (24\%) of those thought not to have TB also had close household contact with adult pulmonary TB.

Table 2. - Historical features and presenting symptoms

\begin{tabular}{lcccc}
\hline & Total & Weight loss & Cough & $\begin{array}{c}\text { Household } \\
\text { contact }\end{array}$ \\
\hline Confirmed TB & 10 & 8 & 5 & 6 \\
Probable TB & 23 & 16 & 8 & 17 \\
No TB & 173 & 133 & 46 & 42 \\
Total & & 157 & 59 & 65 \\
\hline
\end{tabular}

TB: tuberculosis.
Cough and wheeze for $>2$ weeks were reported with similar frequency in all three diagnostic groups and the proportion of children with loss of weight or failure to gain adequate weight did not differ between the groups. It is worth noting that no case of TB was identified in the children with failure to gain weight as a single criterion.

A chest radiograph was taken of 157 children $(76 \%)$ and the findings of 145 children are summarized in table 3 . Six radiographs (4\%) were presumed lost and the quality of six was not satisfactory for evaluation. Based on radiological criteria, 10 children $(7 \%)$ were finally diagnosed as confirmed TB cases and $18(12 \%)$ as probable TB cases and $117(81 \%)$ were considered not to have TB. After evaluation of all chest radiographs, six of the culture-confirmed TB cases $(60 \%)$, nine $(50 \%)$ of the probable TB (based on the criteria in table 1b) and seven (5\%) of the children thought not to have TB in the final analysis were considered as indicative of TB. One of the 10 children with TB confirmed by culture of $M$. tuberculosis from the gastric aspirate had a normal chest radiograph. This child presented with cough accompanied by wheezing for $>2$ weeks. One case showed a miliary pattern supporting the diagnosis of probable TB. Of the children with a positive culture, three had an abnormal chest radiograph, which was not considered indicative of TB by the panel.

Three of the children had not been given a BCG vaccination, but none of them was finally diagnosed as having TB. The other children had received the BCG vaccination neonatally.

A Mantoux test was conducted in 151 children (73\%), but the test was read in only 106 (70\%), of whom 24 (23\%) had a transverse induration $\mathrm{S} 15 \mathrm{~mm}$.

Two children in the study were HIV positive, neither of whom was diagnosed as having TB and both of whom had a negative Mantoux test.

Table 3. - Radiographic features in children with confirmed and probable tuberculosis (TB) and in those thought not to have TB

\begin{tabular}{|c|c|c|c|}
\hline Clinical features & $\begin{array}{c}\text { Confirmed } \\
\text { TB } \\
n=10\end{array}$ & $\begin{array}{c}\text { Probable } \\
\text { TB } \\
n=18\end{array}$ & $\begin{array}{l}\text { Not TB } \\
n=117^{*}\end{array}$ \\
\hline \multicolumn{4}{|l|}{$\begin{array}{l}\text { Unequivocal mediastinal } \\
\text { lymphadenopathy }\end{array}$} \\
\hline Hilar only & 2 & 3 & 0 \\
\hline Hilar and paratracheal & 0 & 2 & 0 \\
\hline Paratracheal only & 1 & 0 & 0 \\
\hline \multicolumn{4}{|l|}{$\begin{array}{l}\text { Uncertain mediastinal } \\
\text { lymphadenopathy }\end{array}$} \\
\hline Hilar only & 2 & 2 & 13 \\
\hline Hilar and paratracheal & 0 & 1 & 0 \\
\hline Paratracheal only & 1 & 0 & 2 \\
\hline \multicolumn{4}{|l|}{ Airway compression } \\
\hline Tracheal & 0 & 0 & 0 \\
\hline Bronchial & 0 & 2 & 0 \\
\hline Airtrapping & 0 & 0 & 6 \\
\hline \multicolumn{4}{|l|}{ Opacification } \\
\hline Lobar & 5 & 7 & 25 \\
\hline Segmental & 1 & 1 & 10 \\
\hline Perihilar & 0 & 2 & 30 \\
\hline Peribronchial & 0 & 0 & 8 \\
\hline Bronchopneumonia & 1 & 1 & 5 \\
\hline Collapse & 3 & 1 & 2 \\
\hline Cavity & 3 & 2 & 1 \\
\hline
\end{tabular}


Table 4. - Anamnestic features and presenting symptoms

\begin{tabular}{lrcccc}
\hline $\begin{array}{l}\text { Anamnestic } \\
\text { and } \\
\text { presenting } \\
\text { symptoms }\end{array}$ & Total & $\begin{array}{c}\text { Confirmed } \\
+ \\
\text { probable }\end{array}$ & Confirmed & Probable No TB \\
\hline W & 100 & 5 & 2 & 3 & 95 \\
C & 17 & 2 & 2 & 0 & 15 \\
H & 19 & 4 & 0 & 4 & 15 \\
W+C & 21 & 3 & 0 & 3 & 18 \\
W+H & 25 & 9 & 3 & 6 & 16 \\
C+H & 5 & 1 & 0 & 1 & 4 \\
W+C+H & 11 & 7 & 3 & 4 & 4 \\
LW+C+H & 4 & 3 & 1 & 2 & 1 \\
\hline
\end{tabular}

TB: tuberculosis; W: weight; C: cough; H: household contact; LW: low weight.

It proved possible to obtain one or more gastric aspirates for $M$. tuberculosis culture from only 99 children (48\%). In 57 children (28\%) two gastric aspirates were obtained. In two of the 10 children from whom a culture of $M$. tuberculosis was obtained the first specimen was positive and in four children both the first and second specimens were positive.

Taking into account the results of gastric aspirate culture for TB, chest radiographs showing unequivocal adenopathy, Mantoux tests giving an induration Š $15 \mathrm{~mm}$ and the findings at 8 weeks follow-up the children were classified as having either confirmed TB (10 children), probable TB ( 23 children) or no TB (173 children).

Table 4 summarizes the final diagnosis in those children presenting with one or more of the diagnostic WHO criteria. Of those presenting with two criteria, most cases of TB were found among the 25 children with the combination of loss of weight or failure to gain weight and contact with an adult with TB. Nine of the 25 children (36\%) had confirmed TB (three cases) or probable TB (six cases). In terms of percentage, the number of TB cases increased to $56 \%$ (five of the nine children) when the criterion of contact with an adult with TB was combined with loss of weight only.

All three WHO criteria were present in 11 children. Seven of these children had confirmed TB (three children) or probable TB (four children). As a result, in this group of 627 children, the positive predictive value of the three WHO criteria when present simultaneously was $63 \%$.

\section{Discussion}

This study shows that a combination of the three WHO criteria for TB has a positive predictive value of $63 \%$ in detecting cases of confirmed or probable TB. No useful value was obtained from the presence of only one or two of the individual criteria.

This study was undertaken in the busy outpatient department of a tertiary care hospital serving an underprivileged community with a high incidence of TB. As such, it provides a realistic view of the likely success of the three clinical criteria frequently suggested for selecting children in whom TB should be suspected. The setting of the study is unusual in the context of underprivileged communities in the developing world, in that chest radio- graphy is not usually so freely available, nor is it always possible to obtain gastric aspirate specimens for culture of TB in peripheral clinics.

Accurate diagnosis of TB is important for the institution of appropriate therapy. In the case of childhood TB the decision to institute therapy will be influenced not only by the diagnostic criteria but also by the age of child and the severity of illness. In this study $60 \%$ of the children with confirmed or probable TB were younger than 1.5 yrs. Age may be of particular importance in the younger infants and children aged $<2$ yrs, since these are the children at great risk of developing severe, life-threatening disseminating forms of TB [12].

The children presenting with confirmed and probable $\mathrm{TB}$, however, had a far higher reported incidence of contact with an adult case of pulmonary TB, i.e., 60 and 74\%, respectively. Children in contact with an adult case of TB are exposed to a considerable risk of infection and disease; however, when the criterion of household contact alone is present, this does not necessarily mean that the child in question is suffering from TB [13]. In combination with one or both of the other criteria, the probability of suffering from TB increases.

No cases of TB were identified amongst the children with failure to gain weight as the sole criterion of suspecting TB, and it would appear that for most screening purposes these children could be ignored for TB diagnosis in the absence of other criteria, symptoms or clinical signs.

In adult practice culture of $M$. tuberculosis is the gold standard against which the diagnosis of TB can be measured. In childhood this is only partially true. Even within secondary and tertiary care institutions the diagnosis of TB in childhood is confirmed by culture of TB from gastric aspirate in only $20-30 \%$ of children [14]. With this in mind we were reluctant to ask all 627 patients who entered the clinic to volunteer for gastric aspirate and all other work-up and concentrated instead on the 206 patients with one or more positive WHO criteria (table 1a). This made it impossible to calculate the sensitivity, specificity and negative predictive value of the WHO criteria.

In conclusion, this study assists in the more precise delineation of the positive predictive value of the proposed World Health Organization approach to the diagnosis of tuberculosis in childhood. The diagnosis of tuberculosis must be more seriously considered when a child presents with three of the criteria. We suggest that this is an appropriate diagnostic process for use in areas of high prevalence and with few facilities which will provide a logical basis for what is, in these circumstances, the art of the possible. In children $<1.5$ yrs of age applying the World Health Organization criteria for suspecting tuberculosis may also be of value in countries with high socioeconomic standards.

\section{References}

1. World Health Organization. WHO tuberculosis programme framework for effective tuberculosis control. WHO/TB/94. Geneva, World Health Organization, p. 179.

2. World Health Organization. Childhood tuberculosis and BCG vaccine: EPI Update Supplement. Geneva, World Health Organization, 1989. 
3. Kochi A. The global tuberculosis situation and the new control strategy of the World Health Organization. Tubercle 1991; 72: 1-6.

4. Kustner HGV. Tuberculosis in the Cape Province. Epidemiol Comments 1991; 18: 3-23.

5. Crofton J, Horne N, Miller F. Clinical tuberculosis. 1st Edn. London, Macmillan Education, 1992; p. 29.

6. World Health Organization. Provisional guidelines for the diagnosis and classification of the EPI target diseases for primary health care, surveillance and special studies. EPI/ GEN/83/4. Geneva, World Health Organization, 1983.

7. Beyers N, Gie RP, Zietsman HL, et al. The use of a geographical information system (GIS) to evaluate the distribution of tuberculosis in a high-incidence community. $S$ Afr Med J 1996; 86: 40-44.

8. Medical Officer of Health. Annual report of the Department of Health Services. Cape Town, Western Cape Regional Services Council, 1991; pp. 44-62.
9. Fourie PB. Patterns of tuberculin hypersensitivity in South Africa. Tubercle 1983; 64: 167-179.

10. Glatthaar E, Kleeberg HH. Evaluation of BCG vaccines and new method of multiple puncture administration. $S$ Afr Med J 1977; 52: 633-638.

11. Swanevelder R. Second national HIV survey of women attending antenatal clinics, South Africa, October/November 1991. Epidemiol Comments 1992; 19: 80-89.

12. Lincoln EM. The value of follow up studies of children with primary tuberculosis. Am Rev Tuberc 1951; 64: 499507.

13. Schaaf HS, Beyers N, Gie RP, et al. Respiratory tuberculosis in children: the diagnostic value of clinical features and special investigations. Pediatr Infect Dis J 1995; 14: 189-194.

14. Starke JR. Modern approach to the diagnosis and treatment in children. Paediatr Clin N Am 1988; 35: 441- 464. 